Julien GoyetTe, «De la difficulté d'hériter en histoire », dans Anne CAUMARTin et Martine-Emmanuelle LAPOINTE (dir.), Filiations intellectuelles dans la littérature québécoise, @ nalyses, automne 2007

\title{
Julien GOYETTE
}

\section{De la difficulté d'hériter en histoire}

Dans Les mots et les choses, Foucault soulignait l'étrange vocation des sciences humaines qui prennent pour objet ce qui est en réalité leur condition de possibilité (p. 375). La chose est particulièrement manifeste lorsque l'on considère l'histoire. Wilhelm Dilthey prétendait, non sans perspicacité, que « [1]a condition première des sciences historiques réside dans le fait que je suis moi-même un être historique, que celui qui étudie l'histoire est aussi celui qui la fait» (Warnke, p. 59). C'est parce qu'il se sait une créature historique, parce qu'il a conscience d'agir sur le cours des événements que l'être humain veut comprendre son histoire. Paradoxalement, c'est aussi parce qu'il sait que c'est l'histoire qui le fait, parce qu'il a la conviction d'appartenir à une histoire qui le dépasse qu'il souhaite élucider les grands mécanismes du développement historique. La situation se complique d'autant dans le cas de l'historien, puisqu'en plus de se faire modeler par l'histoire et de la pétrir à son tour, il l'écrit. Pour lui, l'histoire est à la fois milieu, lieu d'action et mode d'expression.

Gaston Bachelard prétendait que «ce qui dure le plus est ce qui se recommence le mieux»(1989, p. ix). Aucune assertion ne pourrait mieux convenir à la connaissance historique. Celle-ci, comme on sait, est sans cesse revitalisée par un présent inédit. Dans une correspondance, Lionel Groulx écrivait que l'« historien est, de tous les écrivains, celui qui entre le plus promptement au musée des fossiles » (p. 45). Par essence, les livres d'histoire vieillissent prématurément. Personne aujourd'hui ne songerait à se fier aveuglement à l'Histoire de France (Jules Michelet) ou à The Making of the English Working Class (E. P. Thomspon). Hériter en histoire doit forcément vouloir dire autre chose que de récupérer des contenus de circonstance. Les legs et les emprunts doivent se situer sur un autre plan. 
Julien GoyetTe, «De la difficulté d'hériter en histoire », dans Anne CAUMARTin et Martine-Emmanuelle LAPOINTE (dir.), Filiations intellectuelles dans la littérature québécoise, @ nalyses, automne 2007

\section{Entre pensée divergente et pensée convergente}

S'agissant de rappeler l'inéluctable relativité qui s'attache à la pratique historique, il est habituel d'opposer entre elles les différentes générations et cohortes d'historiens afin de faire ressortir les conflits d'interprétations et les percées théoriques et méthodologiques. C'est oublier tout ce que ces généralisations peuvent avoir d'abusif et à quel point les générations sont moins cohérentes qu'il n’y parait. Assoiffé de tendances, on néglige parfois aussi de montrer que la dynamique constitutive de l'histoire est perceptible jusque dans la trajectoire personnelle des historiens. En 1969, l'historien Guy Frégault ouvrait ses propos liminaires de la réédition de La civilisation de la Nouvelle-France en parlant du « jeune homme qui a écrit ce petit livre » comme s'il s'agissait d'un autre que lui. Plus loin (p. 175-177), il avoue qu'après avoir abandonné son œuvre derrière lui pendant 25 ans, il ne sait plus très bien si, maintenant, c'est lui qui la reprend ou si c'est elle qui le ressaisit.

Le relativisme de la connaissance historique ne fait aucun doute. Ce fut la grande œuvre de l'épistémologie contemporaine de révéler, un à un, les nombreux conditionnements qui pèsent sur l'historien. À la fin de cet exercice de démystification, qui fut, il faut l'avouer, un long et douloureux acte d'humilité pour les historiens, l'histoire est apparue à d'aucuns comme un simple propos d'auteur, un "artifice littéraire » constamment dissolu dans la contingence de l'histoire. Aux yeux des relativistes radicaux, il n'y aurait plus de contenus stables, seulement des interprétations qui se succèdent sans jamais parvenir à s'imposer.

Déjà, il est possible de toucher du doigt une première limite de la filiation intellectuelle en histoire : c'est-à-dire que si l'histoire recommence depuis zéro à chaque historien, l'échange intellectuel est ruiné et, avec lui, la possibilité de filiation. Comme le disait l'historien québécois Jean-Paul Bernard, "S'il n'y a pas quelque chose d'objectif qu'on peut se communiquer, ça ne sert à rien de parler. Il n'y a plus de fondement à la communication. » (p. 7) Soulignons que l'organisation actuelle du champ universitaire pousse à l'avant-gardisme, quand ce n'est pas au radicalisme épistémologique. Encouragés par la concurrence ambiante, de nombreux chercheurs ont développé un goût pour la subversion et 
Julien GoyetTE, «De la difficulté d'hériter en histoire », dans Anne CAumartin et Martine-Emmanuelle LAPOINTE (dir.), Filiations intellectuelles dans la littérature québécoise, @ nalyses, automne 2007

éprouvent constamment la «tentation du vide épistémologique », pour paraphraser Fernand Dumont. Il ne faut pas longtemps pour trouver, dans les écrits universitaires, des déclarations incantatoires appelant à toutes sortes de dépassements et de virages théoriques dont la radicalité n'a d'égale que leur difficulté d'application.

En ce sens, les sciences humaines sont d'authentiques produits de la modernité. En tant que modernes, nous aimons l'idée que la pensée recommence avec nous. Comme le révélerait sans doute une étude de la prose utilisée dans les demandes de subventions, le progrès de la connaissance demeure notre but, ne serait-ce que par esprit de conformisme. Spontanément dans notre esprit, "nouveau » rime avec "meilleur» et « révolutionnaire » avec «plus désirable » (Le Goff, p. 44). Comme le fait remarquer Antoine Prost dans ses Douze leçons sur l'histoire, pour ceux qui accèdent actuellement à la discipline, il n'y a plus guère que deux postures valorisantes: celle du novateur et celle du démystificateur. En exagérant sans doute quelque peu, l'historien français fait remarquer que la défense des traditions disciplinaires n’intéresse pour ainsi dire plus personne et que l'opinion se révèle toujours plus disposée à suivre « un critique blasé qu'un simple d'esprit aux robustes convictions» (p. 283). Reconnaissons qu'il est difficile d'espérer accumuler de nos jours du capital, symbolique ou économique, en se présentant comme un « traditionaliste » sur les plans théorique et méthodologique.

Bien sûr, à l'instar de toute activité intellectuelle, la pratique historique exige sa part de créativité et d'ouverture d'esprit, ce que les psychologues appellent un «mode de pensée divergent». Mais l'histoire appelle aussi des formes de consensus, des réseaux d'adhésion, ce que Thomas Kuhn qualifie, en réponse à ces psychologues, de "mode de pensée convergente ». Dans La tension essentielle, le physicien et historien des sciences rappelait que les révolutions scientifiques impliquent presque à chaque fois la tradition. D'évidence, Kuhn avait en tête ici les sciences naturelles, plus spécifiquement la science dite «fondamentale ». Mais ce qu'il dit vaut encore plus, il me semble, pour les sciences humaines. Le lecteur en jugera par lui-même :

[L]es tournants révolutionnaires d'une tradition scientifique sont relativement rares, et de longues périodes de recherche convergente en sont la condition 
Julien GoyetTE, «De la difficulté d'hériter en histoire », dans Anne CAumartin et Martine-Emmanuelle LAPOINTE (dir.), Filiations intellectuelles dans la littérature québécoise, @ nalyses, automne 2007

nécessaire. [...] [S]eules les investigations fermement enracinées dans la tradition scientifique contemporaine ont une chance de briser cette tradition et de donner naissance à une nouvelle. C'est pourquoi je parle d'une «tension essentielle» implicite dans la recherche scientifique. [...] Très souvent, le chercheur accompli doit faire simultanément preuve d'un caractère traditionaliste et iconoclaste.

$[\ldots]$

[L]e savant productif doit être un traditionaliste qui aime à s'adonner à des jeux complexes gouvernés par des règles préétablies, pour être un innovateur efficace qui découvre de nouvelles règles et de nouvelles pièces avec lesquelles il peut continuer à jouer (p. 307 et 320).

On peut illustrer la nécessité des paradigmes en histoire à partir de l'historiographie québécoise. Vers le milieu des années 1970, l'historienne Louise Dechêne a introduit au Québec de nouvelles méthodes inspirées partiellement des Annales en France, avec son maitre ouvrage Habitants et marchands de Montréal au XVIIe siècle. Ce livre pionnier de l'histoire socio-économique au Québec a suscité de nombreuses vocations et inauguré un véritable chantier sur le Québec préindustriel. Encore aujourd'hui, de nombreux historiens québécois, convaincus qu'«[i]l y a encore beaucoup de bonne musique à écrire en ut majeur» (Prost, p. 283), travaillent non pas à renverser ce paradigme, mais à l'améliorer pour ainsi dire de l'intérieur, à en perfectionner les méthodes et à compléter les connaissances qu'on peut espérer en tirer (voir Wien, p. 249-259). On comprend que le changement en histoire, comme en science en général, n'est jamais absolument révolutionnaire ou traditionnel. La rupture implique toujours, à différent degré, une prise en compte du passé et, continuellement, les historiens balancent entre «l'ennui de recommencer » et « la folie de vouloir innover toujours » (Valéry, p. 21).

Fernand Dumont défendait une perspective similaire à celle de Kuhn, mais sur le plan idéologique. En pensant aux intellectuels contestataires de la Révolution tranquille pressés d'évacuer la «Grande noirceur », il écrivait : "En cette matière, on ne saisit que ce dont on a transgressé l'étrangeté, on n'appréhende que ce dont on s'est fait soimême le sujet.» (1995, p. 364) Plusieurs années auparavant, Lionel Groulx avait tenu à rappeler haut et fort que «[n]ulle génération n'a puissance de se commencer absolument à soi-même» (p. 47). C'est 
Julien GoyetTe, «De la difficulté d'hériter en histoire », dans Anne CAUMARTin et Martine-Emmanuelle LAPOINTE (dir.), Filiations intellectuelles dans la littérature québécoise, @ nalyses, automne 2007

dire que l'ignorance ne peut être tenue pour la mère des révolutions scientifiques et idéologiques, pas plus que la tabula rasa.

\section{Vérité et filiation}

Les querelles historiographiques peuvent, bien entendu, être confondues avec des stratégies discursives de légitimation de la part de certaines cohortes d'historiens. Les charges à l'emporte-pièce d'un Lucien Febvre, fondateur des Annales, contre ses vieux maîtres, Seignobos et Monod, sont fameuses et non dépourvues d'opportunisme (voir Noiriel, chap. 8). Selon un schéma commun à presque tous les contextes nationaux et auquel le Québec ne fait pas exception, les historiens ont d'abord été des chercheurs autodidactes et solitaires. Des historiens comme Michelet ou Fustel de Coulanges étaient réputés n'avoir ni maittre ni disciple, ce qui évidemment, du point de vue sociologique, n'était pas rigoureusement exact. La conception de la science d'un Fustel de Coulanges excluait a priori toute forme de division du travail intellectuel. Une division que l'on retrouvera pourtant au fondement de la disciplinarisation de l'histoire à la fin du XIX siècle. Dans une leçon d'ouverture à la Sorbonne, Fustel de Coulanges se livrait à un petit exercice de spéculation :

Supposez cent spécialistes se partageant par lots le passé de la France; croyezvous qu'à la fin ils auront fait l'histoire de la France? J'en doute beaucoup : il leur manquera au moins le lien des faits, or ce lien est aussi une vérité historique. (Hartog, p. 363)

L'historien français trahit ici son héritage romantique. Pour lui, la qualité de l'histoire tient avant tout au génie de celui qui l'écrit. L'historien doit assumer seul l'ensemble des étapes de la production historique, de la collecte des données jusqu'à la synthèse en passant par l'analyse. Non seulement lui faut-il lire lui-même les documents, mais il doit les lire tous, interpréter chaque ligne, chaque mot jusqu'à qu'il renoue avec le sens original du texte. Scrupuleux à l'extrême, Fustel de Coulanges redoutait par-dessus tout l'esprit de spécialisation. Il peinait à accorder sa confiance à ses collègues historiens, qu'il n'hésitait d'ailleurs pas à rabrouer pour leur manque de méthode ou d'objectivité. 
Julien GoyetTe, «De la difficulté d'hériter en histoire », dans Anne CAUMARTin et Martine-Emmanuelle LAPOINTE (dir.), Filiations intellectuelles dans la littérature québécoise, @ nalyses, automne 2007

Une conception aussi exacerbée de la vérité historique devient virtuellement paralysante. Elle conduit à une forme d'impuissance intellectuelle. Dans une lettre à son datant du 14 avril 1874, Fustel de Coulanges confessait à son frère :

Je travaille beaucoup depuis six mois, mais je ne publie rien. J'éprouve une répugnance instinctive à me faire imprimer; mes notes et mes travaux remplissent mes cartons, sans que je puisse me résoudre à rien donner à la Revue des Deux Mondes. Mon grand plaisir est de chercher la vérité, bien plutôt que de la dire. (Hartog, p. 66)

L'auteur de la Cité antique se faisait une conception tellement haute de la vérité qu'il n'osait plus rien écrire de peur de la trahir. À travers ce dernier cas de figure, on atteint une seconde limite de la filiation intellectuelle en histoire : l'excès de doute et de critique finit par tarir les sources de la communication et fait en sorte que l'intellectuel, à la limite, ne peut ni recevoir ni donner intellectuellement. Passé un certain seuil, quand elle ne peut plus être éprouvée que sous la forme d'une aperception, la vérité condamne ses fidèles à la solitude.

J'ai pris un exemple français. J'aurais pu tout aussi bien m’inspirer de l'historiographie québécoise, qui regorge elle aussi de conflits de succession qui font le miel des historiographes et où se mêlent arguments scientifiques et stratégies discursives de légitimation. Au Québec, c'est après la Deuxième Guerre mondiale que l'histoire se disciplinarise, grâce en large part à Lionel Groulx, fondateur de l'Institut d'histoire et de la Revue d'histoire de l'Amérique française. Ce passage de l'histoire dite amatrice à l'histoire professionnelle a donné lieu à l'une des plus importantes ruptures de l'historiographie québécoise. En 1949, Marcel Trudel écrivait: " [...] l'histoire, au Canada français, est toujours confortablement assise dans la chaire de rhétorique et regarde de bien haut l'historienchercheur qui veut être scientifique. » (Bédard et Goyette, p. 151). Si la rupture s'est accomplie dans un respect relatif en ce qui concerne les héritiers directs, les écoles de Montréal (Frégault, Brunet, Séguin) et de Québec (Trudel, Ouellet, Hamelin), il en va autrement pour la génération suivante, celle des historiens des décennies 1960 et 1970. À partir de cette époque, il semble bien que la transition se soit faite davantage dans l'indifférence des anciens que dans une démarche qui allierait critique et 
Julien GoyetTe, «De la difficulté d'hériter en histoire », dans Anne CAUMARTin et Martine-Emmanuelle LAPOINTE (dir.), Filiations intellectuelles dans la littérature québécoise, @ nalyses, automne 2007

réévaluation du travail déjà accompli. Il semblait d'ailleurs à l'historien Jean Hamelin que c'est « une caractéristique de notre tradition québécoise intellectuelle que de fonctionner moins par rupture que par ignorance des devanciers » (Bédard et Goyette, p. 221). Les historiens modernistes n'ont pas rejeté les thèses des anciens; ils ont déserté le chantier pour aller travailler ailleurs. Ils n'ont pas refusé l'héritage; ils ont refusé d'être des héritiers, du moins des héritiers de la tradition locale. En intégrant les problématiques étrangères, principalement françaises et américaines, ces historiens ont eu l'impression d'avoir accompli un saut qualitatif important, ce qui, à bien des égards, était loin d'être faux. Par contre, en n’inscrivant pas toujours leur pratique dans la continuité de ce qui s'était fait auparavant au Québec, ils ont eu tendance à assimiler un simple changement d'objet d'étude à un basculement dans un régime discursif totalement inédit.

\section{Le lieu de la pensée historique}

En histoire, le passage des pouvoirs ne s'effectue donc pas toujours dans la sérénité. Le relativisme des interprétations, le culte de l'originalité et le déni des prédécesseurs viennent souvent brouiller les emprunts et masquer les permanences. Pourtant, à la lumière des propos précédents, on peut se demander s'il n'y a vraiment que du changeant dans les œuvres historiques. Vrai que l'historiographie est traversée d'antagonismes, de conflits d'interprétation et de remises en cause. Mais en deçà de ces oppositions, est-il possible d'apercevoir une certaine stabilité de forme et de contenu? Au fond, le problème de l'histoire est celui de la culture en général. S’interrogeant sur la discontinuité de la pensée moderne, Foucault écrivait: «[...] comment se fait-il que la pensée ait un lieu dans l'espace du monde, qu'elle y ait comme une origine, et qu'elle ne cesse, ici et là, de commencer toujours à nouveau? » (p. 64) Où faut-il chercher le lieu de la pensée historique? Quel est l'enracinement du discours historique qui lui permet de se tenir debout malgré le vent qui rabat inlassablement au sol la parole historienne?

On pense d'abord aux "faits bruts», sur lesquels les historiens s'entendent généralement et qui assurent déjà une stabilité aux interprétations. On pense aussi au style, aux procédés de mise en intrigue et aux 
Julien GoyetTE, «De la difficulté d'hériter en histoire », dans Anne CAumartin et Martine-Emmanuelle LAPOINTE (dir.), Filiations intellectuelles dans la littérature québécoise, @ nalyses, automne 2007

«propositions narratives» sur lesquels insistent les théoriciens de l'histoire depuis une quarantaine d'années (voir Danto et White). Mais il y a davantage. Dans un article datant des années 1960, Fernand Dumont faisait remarquer que tous les points de vue théoriques ne sont pas applicables à l'histoire: "La subjectivité du chercheur ne s'exerce pas seulement sur les "faits", mais aussi par référence à ce que nous appellerions volontiers des "traditions historiographiques". »(1966a, p. 269) La définition que Dumont donne de ces traditions correspond assez bien à celle que l'on associe aux philosophies de l'histoire :

À côté de traditions plus ou moins diffuses, espèces de lieux communs de la conscience historienne, il existe de véritables structures préalables de la perception historique par rapport auxquelles peuvent précisément jouer les variations des points de vue des divers historiens. (1966a, p. 264)

Pour illustrer cette réalité, le sociologue de l'Université Laval évoque les différentes écoles historiques du Canada français qui, tout en s'opposant vigoureusement sur certains points d'histoire, n'en réfèrent pas moins toutes à des schémas de développement communs. Par exemple, jusqu'aux années 1960, les historiens québécois accordaient tous une grande importance à la Conquête, aux luttes politiques qui avaient suivi l'Acte constitutionnel de 1791 et, comme de raison, au génie déployé par les Canadiens français pour survivre dans un contexte hostile. Plus tard, avec l'avènement de la Révolution tranquille, les intellectuels québécois ont développé les récits Conservation/Rattrapage/Développement et sa variante Grande noirceur/Révolution tranquille/Québec moderne. D’un point de vue plus occidental, on peut penser également à la découpe traditionnelle de l'histoire Antiquité-Moyen Âge-Renaissance-Temps modernes qui contient, en filigrane, une indéniable philosophie de l'histoire.

Avant de nous servir ses savantes vérifications, l'historien doit qualifier le mouvement historique; il doit intercaler ses propres récits dans ces grands schémas du devenir que sont les philosophies de l'histoire. Ce que j'ai appelé imprudemment plus haut les « faits bruts » ne tirent en réalité leur valeur et leur signification que de leur insertion dans des structures narratives. Celles-ci servent de toile de fond aux interpréta- 
Julien GoyetTe, «De la difficulté d'hériter en histoire », dans Anne CAUMARTin et Martine-Emmanuelle LAPOINTE (dir.), Filiations intellectuelles dans la littérature québécoise, @ nalyses, automne 2007

tions historiques et permettent, du même coup, une forme de continuité entre les grandes familles d'esprit en histoire.

Dumont nous offre un autre concept, plus audacieux celui-là, permettant de dépasser les oppositions de surface. Dans son article «Le projet d'une histoire de la pensée québécoise », il évoque l'existence d'une mémoire d'intention, d'une «solidarité dans les questions » qui se serait transmise à travers les générations d’intellectuels canadiens-français. Cette mémoire tiendrait à un questionnement maintes fois repris sur la condition de la communauté canadienne-française, sur ses origines, son identité et sa quête d'affranchissement. Il n'est pas abusif, je pense, d'appliquer ce concept de mémoire d'intention à l'historiographie, surtout quand on sait l'importance qu'accordait Dumont à la conscience historique et à ses fondateurs, tels François-Xavier Garneau et Lionel Groulx. Dans une perspective herméneutique, et devant le déni du passé qui accompagne selon lui le discours de la Révolution tranquille, Dumont tenait à réaffirmer l'appartenance fondamentale de la conscience à l'histoire, ou si l'on préfère l'impossibilité de se soustraire entièrement à l'influence du passé. Écrire l'histoire, laisse-t-il entendre après Nietzsche, n'est pas une fin en soi. La volonté d'expliquer le passé est prélevée sur un besoin de comprendre qui prend sa source dans les solidarités concrètes (nationales, idéologiques, disciplinaires, etc.) de l'historien. Selon cette perspective, avant d'être des ensembles de procédures méthodologiques, les disciplines seraient des héritages de questions. «Nous ne demandons pas à Aristote ou à Descartes comment faire de la physique ni à Auguste Comte ou à Proudhon comment pratiquer la sociologie. Nous cherchons chez eux des origines, des impulsions à penser. »(Dumont, 1995, p. 361) Ce que Dumont a dit un jour du philosophe (1986, p. 53), on pourrait le dire de l'historien: on le devient et on le demeure par récapitulation. En plaçant la mémoire au "commencement de la méthode» (Dumont, 1995, p. 363), en inscrivant son travail dans la continuité d'une origine, l'historien sort de sa solitude pour rejoindre une « communauté des interprétants ».

Une indéniable distance sépare les travaux des différentes générations d'historiens. Cette distance, dans une perspective qui était chère encore une fois à Fernand Dumont, nous sommes libres de la voir comme un obstacle à franchir ou comme le chemin qui ramène à 
Julien GoyetTe, «De la difficulté d'hériter en histoire », dans Anne CAUMARTin et Martine-Emmanuelle LAPOINTE (dir.), Filiations intellectuelles dans la littérature québécoise, @ nalyses, automne 2007

l'intention fondamentale de l'écriture historique. Loin d'être un détour inutile, elle serait la voie royale qu'il nous faut suivre pour atteindre le noyau dur de la discipline historique, un moyen de l'inscrire dans la durée, dans sa durée. Dans une pratique qui se maintient dans un perpétuel état de crise, penser par récapitulation constitue une manière de refaire constamment l'unité de la discipline.

\section{Bibliographie}

BACHELARD, Gaston. 1989 [1950], La dialectique de la durée, Paris, Presses Universitaires de France, coll. «Quadrige ».

BÉDArd, Éric et Julien GoyetTE. 2006a, Parole d'historiens. Anthologie des réflexions sur l'histoire au Québec, Montréal, Les Presses de L’Université de Montréal, coll. « Corpus ».

BERNARD, Jean-Paul (invité) et François RICARD (animateur). 1981, Jean-Paul Bernard. 16 août 1981, Montréal, Société Radio-Canada, coll. «Écrire l'histoire au Québec », cahier n ${ }^{\circ} 10$.

DAnto, Arthur. 1968, Analytical Philosophy of History, Cambridge, Cambridge University Press.

DUMONT, Fernand. 1966a. «Idéologie et conscience historique dans la société canadienne-française du XIX ${ }^{\mathrm{e}}$ siècle », dans Claude GALARNEAU et ElZÉAR Lavoie (dir.), France et Canada français du XVI au $X X^{e}$ siècle, Québec, Les Presses de l’Université Laval, p. 269-290;

-. 1986, «Mutations culturelles et philosophie », dans Venant CAuchy (dir.), Philosophie et culture. Actes du XVII Congrès mondial de philosophie, t. I, Montréal, Éditions du Beffroi/Éditions Montmorency, p. 45-55;

-. 1995 [1987], Le sort de la culture. Essai, Montréal, l’Hexagone/Éditions Typo. 
Julien GoyetTe, «De la difficulté d'hériter en histoire », dans Anne CAUMARTin et Martine-Emmanuelle LAPOINTE (dir.), Filiations intellectuelles dans la littérature québécoise, @ nalyses, automne 2007

Foucault, Michel. 1966b, Les mots et les choses, Paris, Éditions Gallimard, coll. « Tel ».

GroulX, Lionel. 1998, Une anthologie, textes choisis et présentés par Julien GoYeTte, Montréal, Bibliothèque québécoise.

HARTOG, François. 2001 [1988], Le XIX siècle. Le cas Fustel de Coulanges, Paris, Éditions du Seuil, coll. « Points Histoire ».

KuHN, Thomas S. 1990 [1977], La tension essentielle: tradition et changement dans les sciences, Paris, Gallimard, coll. «Bibliothèques des sciences humaines ».

LE GoFf, Jacques. 1988, Histoire et mémoire, Paris, Gallimard, coll. «Folio histoire ».

NOIRIEL, Gérard. 1996b, Sur la «crise » de l’histoire, [s.l.], Éditions Belin, coll. « Socio-histoires ».

Prost, Antoine. 1996c, Douze leçons sur l'histoire, Paris, Éditions du Seuil, coll. « Points Histoire ».

VALÉRY, Paul. 1924-1925. Variété I et II, Paris, Gallimard, coll. «Folio. Essais ».

WARNKE, Georgia. 1991, Gadamer, herméneutique, tradition et raison, trad. par Jacques Colson, Bruxelles/Paris, De BoeckWesmael/Éditions universitaires, coll. «Le Point philosophique ».

WHITE, Hayden. 1973, Metahistory: The Historical Imagination in Nineteenth Century Europe, Baltimore, Hopkins University Press.

WIEN, Thomas. 2006b, «Louise Dechêne. Habitants et Marchands de Montréal au XVII siècle », dans Claude CORBO (dir.), Monuments 
Julien GoyetTe, «De la difficulté d'hériter en histoire », dans Anne CAUMARTin et Martine-Emmanuelle LAPOINTE (dir.), Filiations intellectuelles dans la littérature québécoise, @nalyses, automne 2007

intellectuels québécois du XX siècle. Grands livres d'érudition de science et de sagesse, Sillery, Septentrion, p. 249-259. 